\section{Función renal al fallecimiento según causa de muerte en trasplantados renales}

\author{
JUAN PABLO HUIDOBRO E. ${ }^{1,2}$, JORGE VEGA $^{1,2,3}$
}

\section{Glomerular filtration rate before death, in patients who died with a functioning kidney graft}

Background: Death with a functioning kidney graft is now the leading cause of graft loss after renal transplantation. Aim: To determine if renal function at the last outpatient visit before the recipient's death was different according to the following causes of death: infections, cardiovascular diseases, gastrointestinal disorders or cancer. Patients and Methods: Retrospective cooperative cohort study conducted in two kidney transplant centers. All patients who received a kidney graft in the last 32 years were included. During that lapse, 431 kidney transplants were performed. Among them, 85 patients died with a functioning graft and were classified due to their cause of death. Glomerular filtration rate (eGFR) was estimated with the Modification of Diet in Renal Disease formula. Declining renal function was defined as a $>20 \%$ decline in eGFR during the last 6 months prior to the last outpatient visit. Results: Eighty four patients were included in the analysis. Of these, $28.2 \%$ died of cardiovascular causes, $35.3 \%$ of infectious diseases, $15.3 \%$ of cancer and $20 \%$ of digestive diseases. Patients dying from cancer had a significantly higher eGFR prior to death than those who died from cardiovascular causes, infectious and digestive diseases $(p<0.01)$. Declining renal function occurred in $34.8 \%$ of deaths from cardiovascular causes, $39.3 \%$ from infectious diseases, $16.7 \%$ from cancer and $40 \%$ from digestive diseases. There were no significant differences between groups. Conclusions: Kidney graft recipients who die with a functioning graft have better renal function prior to their death when it is due to cancer than when it originates from infectious, cardiovascular or digestive diseases. Declining renal function is similar in the four groups of death causes.

(Rev Med Chile 2016; 144: 853-861)

Key words: Cancer; Infection; Cardiovascular Diseases; Kidney Transplantation; Mortality.

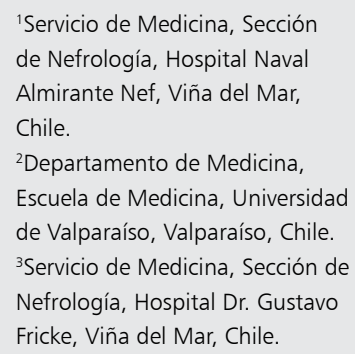

Recibido el 13 de enero de 2016 , aceptado el 29 de junio de 2016.

Correspondencia a:

Dr. Jorge Vega Stieb

5 Norte 1035, Viña del Mar,

Chile.

Teléfono: 56-32-2974237.

jvegastieb@gmail.com
I a enfermedad renal crónica es una patología de prevalencia creciente debido al aumento en las expectativas de vida de la población y de su asociación a enfermedades crónicas no transmisibles ${ }^{1}$.

La sobrevida y calidad de vida de los pacientes sometidos a un trasplante renal (TxR) es superior a la de los que permanecen en diálisis crónica ${ }^{2,3}$.

La muerte con riñón funcionante (MCRF) actualmente es la causa principal de pérdida de los injertos, desplazando al agotamiento de la función del riñón trasplantado con regreso a la terapia dialítica ${ }^{4-6}$. La mejoría de los esquemas de inmunosupresión y del manejo de las enfermeda- 
des crónicas (principalmente cardiovasculares), así como el tratamiento de enfermedades infecciosas han prolongado la duración de los órganos trasplantados ${ }^{7,8}$.

No existe mucha información acerca de si la función renal previa al fallecimiento es diferente según la causa de muerte en los pacientes que fallecen con un riñón funcionante.

El objetivo primario de este estudio fue conocer si la función renal en el último control ambulatorio, previo a la muerte del receptor del injerto, fue diferente si la causa de muerte fue una enfermedad infecciosa, digestiva, cardiovascular o cáncer.

El objetivo secundario fue conocer si una función renal declinante en los 6 meses previos al fallecimiento fue más frecuente en los fallecidos por alguna de estas cuatro causas.

\section{Material y Método}

Entre los años 1981 y 2013, 431 pacientes trasplantados renales fueron controlados en los policlínicos de Nefrología y TxR de los hospitales Dr. Gustavo Fricke de Viña del Mar y Hospital Naval Almirante Nef de Valparaíso y Viña del Mar. De ellos, 85 fallecieron con un riñón funcionante $y$ constituyen los pacientes incluidos en esta investigación, la cual fue aprobada por el Comité Ético-Científico.

Se definió como muerte con riñón funcionante al fallecimiento ocurrido en un trasplantado renal con una velocidad de filtración glomerular suficiente para no requerir de diálisis $(>15 \mathrm{ml} /$ $\mathrm{min} / 1,73 \mathrm{~m}^{2}$ ) y que no presagiaba que retornaría a diálisis crónica en los tres meses siguientes. Se incluyó a todos los pacientes trasplantados de riñón entre 1981 y 2013 que fallecieron con un injerto funcionante y que fueron controlados en los policlínicos de nefrología o trasplante renal de los hospitales Dr. Gustavo Fricke u Hospital Naval en al menos tres oportunidades.

Las causas del fallecimiento se clasificaron en 5 categorías: enfermedades cardiovasculares, infecciones, neoplasias malignas, enfermedades gastrointestinales y misceláneas.

Se registraron los datos demográficos, características inmunológicas en receptores y donantes, etiologías de la enfermedad renal crónica, intervalo entre el diagnóstico de la insuficiencia renal e ingreso a diálisis, tiempo de permanencia en diálisis crónica hasta el TxR, lugar donde se efectuó el TxR, inmunosupresión utilizada, complicaciones ocurridas durante la evolución del TxR, función renal durante el seguimiento y estado de los pacientes e injertos a la fecha de corte de esta investigación (31 de diciembre de 2013).

Se estimó la velocidad de filtración glomerular (eVFG) con la fórmula MDRD de cuatro variables $^{9,10}$.

Se consideró que los pacientes fallecieron con una función renal declinante cuando existió una caída de la eVFG mayor de $20 \%$ entre las estimaciones efectuadas 6 meses antes del último control ambulatorio y el último control previo a la hospitalización en que ocurrió el fallecimiento.

Para el análisis estadístico se utilizó frecuencias, porcentajes, mediana y rango intercuartil, según correspondiera. Dado que cada grupo constaba de un número de observaciones igual o menor a 30 , se asumió una distribución no paramétrica de los datos para cada variable. El análisis multivariado se realizó mediante las pruebas estadísticas de Kruskal-Wallis para variables cuantitativas y test exacto de Fisher para variables cualitativas. Se consideró significativo un valor de $\mathrm{p}<0,05$.

\section{Resultados}

De los 85 pacientes que fallecieron con un riñón funcionante durante un período de observación de 32 años, 30 (35,3\%) fallecieron como consecuencia de una infección, $24(28,2 \%)$ de patologías cardiovasculares, $17(20,0 \%)$ de enfermedades gastrointestinales y $13(15,3 \%)$ de neoplasias malignas. Uno $(1,2 \%)$ falleció en un accidente de tránsito.

Los 84 pacientes fueron categorizados en cuatro grupos, excluyendo al sujeto fallecido accidentalmente.

No hubo diferencias significativas en la edad ni en el género en donantes y receptores entre las 4 categorías, con excepción de los fallecidos por neoplasias malignas, en que fue más frecuente que tuvieran donantes de género masculino $(84,6 \%)$ (Tabla 1).

La proporción de donantes provenientes de sujetos fallecidos fue similar entre los grupos (50\% a $69,2 \%$ ) (Tabla 1).

El intervalo entre el diagnóstico de insuficien- 
Tabla 1. Características demográficas, clínicas e inmunológicas de donantes y de receptores muertos con riñón funcionante

\begin{tabular}{|c|c|c|c|c|c|c|}
\hline \multirow{3}{*}{$\begin{array}{l}\text { Características } \\
\text { Edad receptor, años, mediana (RIC) }\end{array}$} & \multirow[b]{2}{*}{$\begin{array}{c}\text { Cardiovascular } \\
\qquad n=24\end{array}$} & \multicolumn{3}{|c|}{ Mortalidad por causa } & \multirow[b]{2}{*}{$\begin{array}{c}\text { Digestiva } \\
n=17\end{array}$} & \multirow[b]{2}{*}{$\mathbf{p}$} \\
\hline & & $\begin{array}{c}\text { Infecciosa } \\
n=\mathbf{3 0}\end{array}$ & $\begin{array}{r}\text { Neo } \\
\mathbf{n}\end{array}$ & $\begin{array}{l}\text { plásica } \\
=13\end{array}$ & & \\
\hline & $46(32-55)$ & $47(41-51)$ & 51 & $(48-53)$ & $44(36-50)$ & 0,2 \\
\hline Edad donante, años, mediana (RIC) & $32(23-52)$ & $32(23-44)$ & 36 & $(26-47)$ & $37(27-45)$ & 0,8 \\
\hline Sexo donante, \% masculino & $10(45,5 \%)$ & $11(36,7 \%)$ & 11 & $(84,6 \%)$ & $9(52,9 \%)$ & 0,03 \\
\hline Sexo receptor, \% masculino & $12 \quad(50 \%)$ & $17(56,7 \%)$ & 9 & $(69,2 \%)$ & $12(70,6 \%)$ & 0,5 \\
\hline Tipo donante, \% donante fallecido & $12 \quad(50 \%)$ & $19(63,3 \%)$ & 9 & $(69,2 \%)$ & $10(58,8 \%)$ & 0,7 \\
\hline Intervalo IRC-HD, meses, mediana (RIC) & $4 \quad(2-21)$ & $5(2-22)$ & 14,5 & $(6,5-60)$ & $10 \quad(1-20)$ & 0,5 \\
\hline Intervalo HD-TxR, meses, mediana (RIC) & $14,5(6-27)$ & $37(13-64,5)$ & & $5(12-52)$ & $24(11-46)$ & 0,2 \\
\hline Etiología IRC & & & & & & 0,7 \\
\hline Desconocida, n (\%) & $12 \quad(50 \%)$ & $10 \quad(33,3 \%)$ & 4 & $(30,8 \%)$ & $5(29,4 \%)$ & \\
\hline Glomerulonefritis crónica, n (\%) & $7(29,2 \%)$ & $8 \quad(26,7 \%)$ & 6 & $(46,2 \%)$ & $5(29,4 \%)$ & \\
\hline Riñones poliquísticos, n (\%) & 0 & $5 \quad(16,7 \%)$ & 1 & $(7,7 \%)$ & $2(11,8 \%)$ & \\
\hline Pielonefritis crónica, n (\%) & $2(8,3 \%)$ & $4 \quad(13,3 \%)$ & 0 & & $1 \quad(5,9 \%)$ & \\
\hline Nefroesclerosis, n (\%) & 0 & $(3,3 \%)$ & 0 & & $1 \quad(5,9 \%)$ & \\
\hline Nefropatía diabética, n (\%) & $2 \quad(8,3 \%)$ & 0 & 1 & $(7,7 \%)$ & $1 \quad(5,9 \%)$ & \\
\hline Nefropatía lúpica, n (\%) & $1 \quad(4,2 \%)$ & $(3,3 \%)$ & 1 & $(7,7 \%)$ & $1 \quad(5,9 \%)$ & \\
\hline Otras, n (\%) & 0 & $1 \quad(3,3 \%)$ & 0 & & $1 \quad(5,9 \%)$ & \\
\hline Serología (+) virus C de hepatitis, n (\%) & $1 \quad(4,2 \%)$ & $5 \quad(16,7 \%)$ & 0 & & $4(23,5 \%)$ & 0,1 \\
\hline Serología (+) virus B de hepatitis, n (\%) & 0 & $(10 \%)$ & 1 & $(7,7 \%)$ & $2(11,8 \%)$ & 0,3 \\
\hline Diabetes mellitus previa al TxR, n (\%) & 3 & 0 & 1 & & 1 & 0,2 \\
\hline \multicolumn{7}{|l|}{ Inmunología } \\
\hline PRA máximo, \%, mediana (RIC) & $3(0-21)$ & $(0-17)$ & 5 & $(0-8)$ & $8(1,25-24)$ & 0,7 \\
\hline PRA pre-TxR, \%, mediana (RIC) & $(0-5)$ & $(0-0)$ & 0 & $(0-5)$ & $0(0-4)$ & 0,9 \\
\hline Incompatibilidades HLA AB, mediana (RIC) & $(1-2)$ & $(1-2)$ & 1 & $(1-2)$ & $2(1-2,5)$ & 0,3 \\
\hline Incompatibilidades HLA DR, mediana (RIC) & $1 \quad(0,5-1)$ & $1(0-1)$ & & $0(0-1)$ & $1(0-1)$ & 0,6 \\
\hline
\end{tabular}

RIC: rango intercuartil; IRC-HD: intervalo entre diagnóstico de insuficiencia renal e inicio de diálisis; HD-TxR: intervalo entre inicio de diálisis y trasplante renal; IRC: insuficiencia renal crónica; PRA: anticuerpos reactivos contra linfocitos de panel; HLA AB: antígenos de histocompatibilidad de clase 1 A y B; HLA DR: antígenos de histocompatibilidad clase 2 DR.

cia renal crónica y el ingreso a diálisis (4,0-14,5 meses), así como el intervalo entre el inicio de terapia dialítica y el trasplante renal (14,5-37 meses), no fue diferente entre los grupos (Tabla 1).

En una proporción importante de los pacientes la etiología de la insuficiencia renal crónica fue desconocida $(36,9 \%)$. Cuando ésta pudo establecerse, la glomerulonefritis crónica primaria $(30,9 \%)$ y la enfermedad renal poliquística autosómica dominante $(9,5 \%)$ fueron las etiologías más frecuentes de la falla renal. La nefropatía diabética fue la causa de la falla renal sólo en 4 pacientes $(4,8 \%)$ (Tabla 1).

La prevalencia de serología positiva para los virus $\mathrm{B}$ y $\mathrm{C}$ de la hepatitis, así como la existencia de diabetes mellitus previa al trasplante, fue similar entre los grupos (Tabla 1).

El número de incompatibilidades HLA, tanto en antígenos de clase I como de clase II, no fue diferente entre los grupos. Tampoco hubo dife- 
rencias en la tasa de anticuerpos reactivos contra linfocitos de panel (PRA) previo al trasplante o en su tasa máxima histórica (Tabla 1).

El esquema inmunosupresor más frecuentemente utilizado después del trasplante fue la asociación de azatioprina, prednisona y un agente anticalcineurínico $(64,3 \%)$, mayoritariamente ciclosporina. Treinta por ciento de los pacientes utilizaron un esquema bi-asociado con azatioprina y prednisona (Tabla 2).

El porcentaje de los pacientes que recibió inducción con anticuerpos antilinfocíticos o anti interleukina 2 fue bajo y no hubo diferencias significativas entre los grupos.

La proporción de pacientes que recibieron terapia antirechazo durante el primer año del trasplante fue similar entre los grupos ( 23 a $53 \%$ ). En los cuatro años siguientes, una proporción menor a 20\% de los pacientes recibió terapia antirechazo, con excepción de los fallecidos por infecciones, en que $42 \%$ recibió dicha terapia (Tabla 2 ).
Al momento del fallecimiento, dos tercios de los pacientes recibían un esquema inmunosupresor que incluía un anticalcineurínico (Tabla 2).

La proporción de pacientes que tuvieron una función retardada del injerto renal fue similar entre los grupos (17 a 35\%). También lo fue la aparición de diabetes mellitus post trasplante $(7,7$ a $20,8 \%$ ) o eritrocitosis que requirió de sangrías o terapia médica $(12,5$ a $17,7 \%)$.

No hubo diferencias en la incidencia de infecciones por virus herpes (varicela zoster, citomegalovirus) o bacilo de Koch entre los grupos. Tampoco las hubo en la incidencia de crisis de gota, fracturas óseas o necrosis ósea avascular (Tabla 3).

La prevalencia de daño hepático crónico fue mayor en los pacientes que fallecieron de una causa digestiva $(23,5 \%)$. Las otras causas de muerte de origen digestivo fueron: pancreatitis aguda, diarreas crónicas y peritonitis por rotura de víscera hueca.

Tabla 2. Inmunosupresión utilizada durante el trasplante de pacientes fallecidos con riñón funcionante

\begin{tabular}{|c|c|c|c|c|c|}
\hline Características & $\begin{array}{l}\text { Cardiovascular } \\
\qquad n=24\end{array}$ & $\begin{array}{c}\text { Mortalidad } \\
\text { Infecciosa } \\
\mathbf{n}=\mathbf{3 0}\end{array}$ & $\begin{array}{l}\text { Neoplásica } \\
\qquad n=13\end{array}$ & $\begin{array}{c}\text { Digestiva } \\
n=17\end{array}$ & $\mathbf{p}$ \\
\hline Esquema inmunosupresión inicial & & & & & 0,91 \\
\hline Azatioprina-Prednisona, n (\%) & $7(29,2 \%)$ & $8(26,7 \%)$ & $4(30,8 \%)$ & $6(35,3 \%)$ & \\
\hline Azatioprina-Prednisona-Anticalcineurínicos, n (\%) & $13(54,2 \%)$ & $21(70 \%)$ & $9(69,2 \%)$ & $11(64,7 \%)$ & \\
\hline MMF-Prednisona-Anticalcineurínicos, n (\%) & $2(8,3 \%)$ & $1 \quad(3,3 \%)$ & 0 & 0 & \\
\hline Anticalcineurínicos-Prednisona, n (\%) & $1 \quad(4,2 \%)$ & 0 & 0 & 0 & \\
\hline Otro & $1 \quad(4,2 \%)$ & 0 & 0 & 0 & \\
\hline Inducción con anticuerpos n (\%) & $1 \quad(4,2 \%)$ & $2(6,7 \%)$ & 0 & 0 & 0,87 \\
\hline TAR durante el primer año del TxR, n (\%) & $10(41,7 \%)$ & $14(46,7 \%)$ & $3(23,1 \%)$ & $9(52,9 \%)$ & 0,4 \\
\hline TAR entre años 2 a 5 post TxR, n (\%) & $4 \quad(20 \%)$ & $10(41,7 \%)$ & $1 \quad(7,7 \%)$ & $1 \quad(7,7 \%)$ & 0,05 \\
\hline Último esquema inmunosupresor utilizado & & & & & 0,1 \\
\hline Azatioprina-Prednisona, n (\%) & $5(20,8 \%)$ & $5(16,7 \%)$ & $3(25 \%)$ & $5(29,4 \%)$ & \\
\hline Azatioprina-Prednisona-Anticalcineurínicos, n (\%) & $5(20,8 \%)$ & $7(23,3 \%)$ & $5(41,7 \%)$ & $11(64,7 \%)$ & \\
\hline mTOR-Prednisona-Anticalcineurínicos, n (\%) & 0 & $1 \quad(3,3 \%)$ & 0 & 0 & \\
\hline Anticalcineurínicos-Prednisona, n (\%) & $7(29,2 \%)$ & $9 \quad(30 \%)$ & $2(16,7 \%)$ & 0 & \\
\hline Prednisona-MMF-Anticalcineurínicos, n (\%) & $4(16,7 \%)$ & $5(16,7 \%)$ & 0 & $1 \quad(5,9 \%)$ & \\
\hline Prednisona-MMF-mTOR, n (\%) & 0 & $2(6,7 \%)$ & $1 \quad(8,3 \%)$ & 0 & \\
\hline Prednisona-MMF, n (\%) & $2(8,3 \%)$ & 0 & 0 & 0 & \\
\hline mTOR-Prednisona, n (\%) & $1 \quad(4,2 \%)$ & $1 \quad(3,3 \%)$ & 0 & 0 & \\
\hline
\end{tabular}

TAR: terapia anti-rechazo; TxR: trasplante renal; MMF: micofenolato mofetil; mTOR: mammalian target of rapamycin 
Tabla 3. Complicaciones del trasplante renal en pacientes fallecidos con riñón funcionante

\begin{tabular}{|c|c|c|c|c|c|}
\hline Complicaciones & $\begin{array}{c}\text { Cardio- } \\
\text { vascular } \\
n=24\end{array}$ & $\begin{array}{c}\text { Mortalida } \\
\text { Infecciosa } \\
\text { n }=\mathbf{3 0}\end{array}$ & $\begin{array}{l}\text { d por causa } \\
\text { Neoplásica } \\
n=13\end{array}$ & $\begin{array}{l}\text { Digestiva } \\
\qquad n=17\end{array}$ & $\mathbf{p}$ \\
\hline Función renal retardada, n (\%) & $4(17,4 \%)$ & $9 \quad(30 \%)$ & $2(16,7 \%)$ & $6(35,3 \%)$ & 0,52 \\
\hline Hospitalizaciones por infecciones en primeros 5 años, n (\%) & $7(30,4 \%)$ & $13(43,4 \%)$ & $4(30,8 \%)$ & $4(23,5 \%)$ & 0,57 \\
\hline Enfermedades cardiovasculares en primeros 5 años, n (\%) & $9(39,1 \%)$ & $3 \quad(10 \%)$ & 0 & $1(6,3 \%)$ & 0,005 \\
\hline Neoplasias malignas en primeros 5 años, n (\%) & $1(4,4 \%)$ & $2(6,7 \%)$ & $5(38,5 \%)$ & $2(12,5 \%)$ & 0,025 \\
\hline Diabetes mellitus post $T \times R, n(\%)$ & $5(20,8 \%)$ & $5(16,7 \%)$ & $1 \quad(7,7 \%)$ & $2(11,8 \%)$ & 0,79 \\
\hline Daño hepático crónico post TxR, n (\%) & 0 & $4(13,3 \%)$ & 0 & $4(23,5 \%)$ & 0,04 \\
\hline Alcoholismo post TxR, n (\%) & $1(4,2 \%)$ & $2(6,7 \%)$ & $1 \quad(7,7 \%)$ & $3(17,7 \%)$ & 0,51 \\
\hline Eritrocitosis post $T x R, n(\%)$ & $3(12,5 \%)$ & $5(16,7 \%)$ & $2(15,4 \%)$ & $3(17,7 \%)$ & 0,95 \\
\hline Infección por virus varicela zoster, n (\%) & $3(12,5 \%)$ & $6(20 \%)$ & $4(30,8 \%)$ & $1 \quad(5,9 \%)$ & 0,31 \\
\hline Infección por citomegalovirus, n (\%) & $3(12,5 \%)$ & $2(6,7 \%)$ & $2(15,4 \%)$ & $1 \quad(5,9 \%)$ & 0,78 \\
\hline Tuberculosis, n (\%) & $1 \quad(4,2 \%)$ & 0 & $1 \quad(7,7 \%)$ & $1 \quad(5,9 \%)$ & 0,37 \\
\hline Fracturas óseas, n (\%) & $4(16,7 \%)$ & $4(13,3 \%)$ & $1 \quad(7,7 \%)$ & $3(17,7 \%)$ & 0,89 \\
\hline Necrosis ósea avascular, n (\%) & $2(8,3 \%)$ & $3 \quad(10 \%)$ & 0 & 0 & 0,53 \\
\hline Gota aguda, n (\%) & $1(4,2 \%)$ & $4(13,3 \%)$ & 0 & $4(23,5 \%)$ & 0,15 \\
\hline Hospitalizaciones por infecciones después del $5^{\circ}$ año, n (\%) & $8 \quad(50 \%)$ & $12(66,7 \%)$ & $3(33,3 \%)$ & $4(57,1 \%)$ & 0,44 \\
\hline Enfermedades cardiovasculares después del $5^{\circ}$ año, n (\%) & $7(43,8 \%)$ & $3(16,7 \%)$ & $1(11,1 \%)$ & $2(28,6 \%)$ & 0,25 \\
\hline Neoplasias malignas después del $5^{\circ}$ año, n (\%) & $1(12,5 \%)$ & $4(22,2 \%)$ & $9(100 \%)$ & $2(28,6 \%)$ & 0 \\
\hline
\end{tabular}

TxR: trasplante renal.

Durante los primeros 5 años del trasplante, la frecuencia de hospitalizaciones originadas por infecciones no fue diferente entre los grupos $(23,5$ a $43,4 \%)$. Sin embargo, las hospitalizaciones motivadas por las enfermedades cardiovasculares y neoplasias fueron más frecuentes en los pacientes que fallecieron por la misma causa de hospitalización (Tabla 3).

La concentración de creatinina sérica al momento del alta de la hospitalización por el trasplante $(1,3$ a $1,6 \mathrm{mg} / \mathrm{dl})$, así como la velocidad de filtración glomerular estimada durante los primeros 10 años de la operación, no fue diferente entre los grupos (Tabla 4).

En el último control ambulatorio previo a la hospitalización que culminó en el fallecimiento, la eVFG fue significativamente superior $(53 \mathrm{ml} /$ $\mathrm{min} / 1,73 \mathrm{~m}^{2}$ ) en los pacientes que fallecieron como consecuencia de una neoplasia maligna que los que fallecieron a consecuencias de una infección $\left(33 \mathrm{ml} / \mathrm{min} / 1,73 \mathrm{~m}^{2}\right)$, patología cardiovascular $\left(30,5 \mathrm{ml} / \mathrm{min} / 1,73 \mathrm{~m}^{2}\right)$ o patologías digestivas $\left(38 \mathrm{ml} / \mathrm{min} / 1,73 \mathrm{~m}^{2}\right)$.

Al considerar la proporción de pacientes que tenían una función renal declinante no se observó diferencias entre los grupos (16,7 a 40,0\%) (Tabla 4).

Tanto la edad al momento del fallecimiento como la duración del TxR no fueron diferentes entre los cuatro grupos (Tabla 4).

\section{Discusión}

En nuestra serie, los pacientes que fallecieron por causa neoplásica lo hicieron con una creatinina menor y una eVFG mayor a los que fallecieron por enfermedades digestivas, cardiovasculares o infecciosas. La proporción de pacientes que tuvo una función renal declinante en los seis meses 
Tabla 4. Función renal durante el trasplante en pacientes fallecidos con riñón funcionante

\begin{tabular}{|c|c|c|c|c|c|}
\hline Función renal & $\begin{array}{l}\text { Cardiovascular } \\
\qquad n=24\end{array}$ & $\begin{array}{c}\text { Mortalidad p } \\
\text { Infecciosa } \\
n=30\end{array}$ & $\begin{array}{l}\text { oor causa } \\
\text { Neoplásica } \\
n=13\end{array}$ & $\begin{array}{l}\text { Digestiva } \\
n=17\end{array}$ & $\mathbf{p}$ \\
\hline $\begin{array}{l}\text { Creatininemia al alta del TxR, mg/dL, } \\
\text { mediana (RIC) }\end{array}$ & $1,6(1,2-2,1)$ & $1,6(1,3-2,2)$ & $1,3(1,2-1,4)$ & $1,5(1,3-1,8)$ & 0,3 \\
\hline $\begin{array}{l}\text { Creatininemia al año } 1 \text { del TxR, mg/dL, } \\
\text { mediana (RIC) }\end{array}$ & $1,3(1,02-1,5)$ & $1,3(1,05-1,7)$ & $1,3(1-1,4)$ & $1,3(1,2-1,8)$ & 0,84 \\
\hline $\begin{array}{l}\text { eVFG al año } 1\left(\mathrm{ml} / \mathrm{min} / 1,73 \mathrm{~m}^{2}\right) \\
\text { mediana (RIC) }\end{array}$ & $56,5(48,5-68,5)$ & $56(39-76)$ & $64(50-75)$ & $65(47-73)$ & 0,83 \\
\hline $\begin{array}{l}\text { Creatininemia al año } 3 \text { del TxR, mg/dL, } \\
\text { mediana (RIC) }\end{array}$ & $1,4(1,1-1,78)$ & $1,3(1,2-1,6)$ & $1,2(1-1,8)$ & $1,6(1,3-2)$ & 0,44 \\
\hline $\begin{array}{l}\text { eVFG al año } 3\left(\mathrm{ml} / \mathrm{min} / 1,73 \mathrm{~m}^{2}\right) \\
\text { mediana (RIC) }\end{array}$ & $52(40-67)$ & $58,5(42-65,5)$ & $66(46-71,5)$ & $48(39-61)$ & 0,58 \\
\hline $\begin{array}{l}\text { Creatininemia al año } 5 \text { del TxR, mg/dL, } \\
\text { mediana (RIC) }\end{array}$ & $1,4(1,3-1,9)$ & $1,6(1,2-2,1)$ & $1,5(1-1,7)$ & $1,4(1,3-1,7)$ & 0,93 \\
\hline $\begin{array}{l}\text { eVFG al año } 5\left(\mathrm{ml} / \mathrm{min} / 1,73 \mathrm{~m}^{2}\right) \\
\text { mediana (RIC) }\end{array}$ & $44(38-60)$ & $53(26-70)$ & $58(43-82)$ & $52(45-60)$ & 0,82 \\
\hline $\begin{array}{l}\text { Creatininemia al año } 10 \text { del TxR, mg/dL, } \\
\text { mediana (RIC) }\end{array}$ & $1,6(1,3-1,9)$ & $1,7(1,3-2,3)$ & $1,1(0,9-1,4)$ & $1,3(1,3-1,6)$ & 0,1 \\
\hline $\begin{array}{l}\text { eVFG al año } 10\left(\mathrm{ml} / \mathrm{min} / 1,73 \mathrm{~m}^{2}\right) \\
\text { mediana }(\mathrm{RIC})\end{array}$ & $43,5(31-52,5)$ & $48(29-55)$ & $71(57,5-97,5)$ & $53(44,5-60,5)$ & 0,14 \\
\hline $\begin{array}{l}\text { Última creatininemia en control ambulatorio } \\
(\mathrm{mg} / \mathrm{dL}) \text {, mediana (RIC) }\end{array}$ & $1,9(1,46-2,81)$ & $2,1(1,6-3,3)$ & $1,2(1-1,5)$ & $1,9(1,6-3,1)$ & 0,005 \\
\hline $\begin{array}{l}\text { Última eVFG en control ambulatorio } \\
\left(\mathrm{ml} / \mathrm{min} / 1,73 \mathrm{~m}^{2}\right) \text {, mediana (RIC) }\end{array}$ & $30,5(20-50,5)$ & $33(17-46)$ & $53(46,5-71)$ & $38(23-44)$ & 0,009 \\
\hline $\begin{array}{l}\text { Duración del TxR (meses), } \\
\text { mediana (RIC) }\end{array}$ & $112(51,5-164,5)$ & $87,5(19-141)$ & $102(53-130)$ & $64(16-104)$ & 0,44 \\
\hline $\begin{array}{l}\text { Edad al fallecimiento (años), } \\
\text { mediana (RIC) }\end{array}$ & $53,5(44-63,5)$ & $55,5(47-62)$ & $62(57-64)$ & $51(45-57)$ & 0,06 \\
\hline $\begin{array}{l}\text { Caída }>20 \% \text { de la eVFG en los últimos } \\
6 \text { meses, n (\%) }\end{array}$ & $8(34,8 \%)$ & $11(39,3 \%)$ & $2(16,7 \%)$ & $6(40 \%)$ & 0,56 \\
\hline
\end{tabular}

TxR: trasplante renal; RIC: rango intercuartil; eVFG: velocidad de filtración glomerular estimada.

previo al fallecimiento fue menor en el grupo fallecido por causa neoplásica, pero no alcanzó diferencias significativas con el resto de los grupos, probablemente por falta de poder estadístico en la serie. Esto sería concordante con las comunicaciones que relacionan la disfunción renal con el riesgo de infecciones severas y complicaciones cardiovasculares.

La muerte con riñón funcionante es la causa de pérdida del injerto hasta en $42,5 \%$ de los pacientes y ocurre entre 9 y $30 \%$ de los receptores ${ }^{4,11}$. El riesgo de MCRF y sus causas han cambiado en las últimas décadas, lo que se ha atribuido a avances en inmunosupresión y disponibilidad de nuevas drogas y tratamientos para los factores de riesgo clásicos de mortalidad cardiovascular, como la hipertensión arterial y la dislipidemia ${ }^{5}$.

En la serie más grande de trasplantados renales publicada hasta este momento (86.502 pacientes), la causa principal de MCRF fue cardiovascular, con $42 \%$ de los casos, seguida de infecciones (17\%), neoplasias (9\%) y enfermedades digestivas $(2 \%)^{5}$. Otras series han comunicado que las infecciones son la principal causa de muerte (hasta 39,7\%), seguido de las enfermedades cardiovasculares ${ }^{5,12}$. Los factores de riesgo de MCRF son: mayor edad del receptor al momento del trasplante ${ }^{2,13}$, rechazo agudo post trasplante ${ }^{2}$, función renal retardada ${ }^{14}$, mal control de la presión arterial, anemia y que la enfermedad renal que motivó el trasplante haya 
sido diabetes ${ }^{13}$. En nuestra serie, la proporción de trasplantados cuya causa de la falla renal fue la diabetes fue muy baja, lo que puede haber tenido influencia en nuestros resultados.

Si bien algunas series reportan que las causas de mortalidad no han variado durante los últimos años $^{12}$, otras reportan que la mortalidad cardiovascular y la debida a neoplasias han aumentado su importancia relativa, disminuyendo las infecciones $^{2,15}$.

Los pacientes trasplantados renales tienen un riesgo de mortalidad por causa cardiaca hasta 10 veces mayor que la población general y una tasa anual de eventos cardiovasculares no fatales al año 50 veces mayor ${ }^{16}$. El riesgo de sufrir un accidente cerebrovascular isquémico o hemorrágico también es superior al de la población general ${ }^{17}$.

La mortalidad cardiovascular se debe principalmente a enfermedad coronaria, correspondiendo a cerca de la mitad de los casos. 5 . Las enfermedades cardiovasculares son actualmente la causa más frecuente de MCRF, aumentando su importancia relativa en las muertes ocurridas en los primeros 30 días del trasplante ${ }^{5}$.

Los factores de riesgo para su ocurrencia son los mismos que en la población general: diabetes mellitus, hipertensión y dislipidemia, a los que se agregan anemia e hipoalbuminemia ${ }^{13}$ y factores asociados al trasplante, como son el rechazo agudo, función renal retardada y duración de la enfermedad renal previa al trasplante ${ }^{18}$.

El deterioro en la función renal se ha asociado a un aumento en el riesgo de mortalidad cardiovascular en la población general ${ }^{19}$. Del mismo modo, en los trasplantados renales se ha demostrado que el deterioro de la función renal al año del trasplante confiere un mayor riesgo de mortalidad cardiovascular ${ }^{20}$. Se postula que la disfunción renal puede causar o exacerbar los factores de riesgo clásicos para la enfermedad cardiovascular, como la hipertensión arterial, dislipidemia, hiperglicemia e hipertrofia ventricular izquierda ${ }^{21}$. En población general, en tanto, la asociación entre el deterioro de la función renal y mortalidad cardiovascular se ha atribuido a ateroesclerosis y problemas crónicos en el manejo de volumen ${ }^{19}$. El tratamiento inmunosupresor utilizado en trasplante renal se ha asociado a la producción o exacerbación de los factores de riesgo clásicos y a un deterioro en la función renal $^{22}$.

Se ha planteado que un seguimiento de los pacientes trasplantados renales mediante sistemas integrados de salud, con retroalimentación basada en sistemas informáticos, podría disminuir la mortalidad cardiovascular mediante un mejor control de los factores de riesgo ${ }^{13}$.

Las infecciones son la segunda causa de MCRF en la mayoría de las series ${ }^{5,13}$, aunque en esta serie fue la primera. Esto podría deberse a la menor edad de los trasplantados, uso de dosis elevadas de corticoides para la terapia antirrechazo (especialmente en pacientes con el esquema inmunosupresor más débil: azatioprina y corticoides) y a un menor tiempo de permanencia en diálisis crónica previo al trasplante. En otras series de países en vías de desarrollo, en que las infecciones son la primera causa de MCRF, se ha planteado que las malas condiciones de higiene y retardo en la consulta al momento de sufrir infecciones contribuyen a la mortalidad por esta causa ${ }^{23}$. Se ha reportado que desde la década 1960-69 hasta ahora las infecciones han disminuido como causa de MCRF.

Las infecciones ocurren con más frecuencia durante el primer año post trasplante, lo que se ha atribuido a la inmunosupresión más potente durante los primeros meses, la terapia antirrechazo y como complicación de la cirugía ${ }^{2,5,15}$.

El deterioro de la función renal y el rechazo agudo también se han asociado a mayor riesgo de infección severa ${ }^{24}$. Esto es concordante con lo observado en nuestra serie, en que los pacientes que fallecieron por causa infecciosa recibieron más frecuentemente terapia antirrechazo después del primer año.

Los trasplantados renales tienen un riesgo de cáncer entre 3 y 5 veces mayor que el de la población general. Las neoplasias corresponden a la causa de muerte con riñón funcionante en 9 a $28 \%$ de los pacientes ${ }^{5,25}$. Ellas representan menos de $5 \%$ de los casos durante el primer año post trasplante, aumentando a $23,7 \%$ de los casos en las MCRF durante el seguimiento. Las neoplasias hematológicas (ej: linfomas) corresponden hasta $13,4 \%$ de las muertes de este grupo ${ }^{5}$.

La MCRF por neoplasias ha aumentado, lo que se ha atribuido a la mayor edad de los receptores de un injerto, a un mayor lapso de tiempo hasta la MCRF y al uso de inmunosupresores más potentes $^{26}$.

La función renal deteriorada se ha asociado a un mayor riesgo de fallecer con un injerto 
funcionante ${ }^{23}$. Un análisis retrospectivo de 256 trasplantados renales que fallecieron con trasplante funcionante demostró que esta asociación era progresiva, con un hazard ratio (HR) de 2,26 por cada $10 \mathrm{~mL} / \mathrm{min} / 1,73 \mathrm{~m}^{2}$ de deterioro de la velocidad de filtración glomerular bajo $40 \mathrm{ml} /$ $\mathrm{min} / 1,73 \mathrm{~m}^{2}{ }^{27}$. Otro estudio demostró que la disfunción renal en las etapas 4 y 5 de enfermedad renal crónica confería un HR 1,58 y 2,8 de MCRF, respectivamente, usando la ERC etapa 2 como referencia ${ }^{28}$. En población general, en tanto, un análisis retrospectivo de 81.064 pacientes fallecidos clasificados según causa de muerte demostró que la proporción de fallecidos por neoplasias disminuía a medida que declinaba la función renal, mientras que la proporción que fallecía por enfermedad cardiovascular o infecciones aumentaba ${ }^{19}$.

En suma, los trasplantados renales que fallecen por neoplasias tienen una velocidad de filtración glomerular, previo al fallecimiento, superior a los pacientes que mueren por infecciones o enfermedades cardiovasculares.

\section{Referencias}

1. Levey AS, Coresh J. Chronic kidney disease. Lancet 2012; 379 (9811): 165-80.

2. El-Agroudy AE, Bakr MA, Shehab El-Dein AB, Ghoneim MA. Death with functioning graft in living donor kidney transplantation: analysis of risk factors. Am. J. Nephrol 2003; 23 (3): 186-93.

3. Wolfe RA, Ashby VB, Milford EL, Ojo AO, Ettenger RE, Agodoa LY, et al. Comparison of mortality in all patients on dialysis, patients on dialysis awaiting transplantation, and recipients of a first cadaveric transplant. N. Engl. J. Med 1999; 341 (23): 1725-30.

4. Hirata M, Cho YW, Cecka JM, Terasaki PI. Patient death after renal transplantation--an analysis of its role in graft outcome. Transplantation 1996; 61 (10): 1479-83.

5. Ojo AO, Hanson JA, Wolfe RA, Leichtman AB, Agodoa LY, Port FK. Long-term survival in renal transplant recipients with graft function. Kidney Int 2000; 57 (1): 307-13.

6. Morales JM, Marcén R, del Castillo D, Andres A, González-Molina M, Oppenheimer F, et al. Risk factors for graft loss and mortality after renal transplantation according to recipient age: a prospective multicentre study. Nephrol. Dial. Transplant 2012; 27 Suppl 4: iv3946.

7. Gómez EG, Hernández JPC, López FJM, García JR,
Montemayor VG, Curado FA, et al. Long-term allograft survival after kidney transplantation. Transplant. Proc 2013; 45 (10): 3599-602.

8. Sood P, Zhu YR, Cohen EP. Death with functioning kidney transplant: an obituarial analysis. Int. Urol. Nephrol 2010; 42 (4): 929-34.

9. Levey AS, Bosch JP, Lewis JB, Greene T, Rogers N, Roth D. A more accurate method to estimate glomerular filtration rate from serum creatinine: A new prediction equation. Ann Intern Med 1999; 130 (6): 461-70.

10. Vickery S, Stevens PE, Dalton RN, van Lente F, Lamb EJ. Does the ID-MS traceable MDRD equation work and is it suitable for use with compensated Jaffe and enzymatic creatinine assays? Nephrol. Dial. Transplant 2006; 21 (9): 2439-45.

11. West M, Sutherland DE, Matas AJ. Kidney transplant recipients who die with functioning grafts: serum creatinine level and cause of death. Transplantation 1996; 62 (7): 1029-30.

12. Vega J, Videla C, Borja H, Goecke H, Martínez F, Betancour P. [Causes of death with a functioning graft among kidney allograft recipients]. Rev Med Chile 2012; 140 (3): 295-304.

13. Kahwaji J, Bunnapradist S, Hsu J-W, Idroos ML, Dudek R. Cause of death with graft function among renal transplant recipients in an integrated healthcare system. Transplantation 2011; 91 (2): 225-30.

14. Narayanan R, Cardella CJ, Cattran DC, Cole EH, Tickam KJ, Schiff J, et al. Delayed graft function and the risk of death with graft function in living donor kidney transplant recipients. Am J Kidney Dis 2010; 56 (5): 961-70.

15. Shimmura $H$, Tanabe $K$, Tokumoto $T$, Ishida H, Ishikawa N, Miyamoto N, et al. Analysis of cause of death with a functioning graft: a single-center experience. Transplant Proc 2004; 36 (7): 2026-9.

16. Foley RN, Parfrey PS, Sarnak MJ. Clinical epidemiology of cardiovascular disease in chronic renal disease. Am J Kidney Dis 1998; 32 (5 Suppl 3): S112-9.

17. Abedini S, Holme I, Fellström B, Jardine A, Cole E, Maes B, et al. Cerebrovascular events in renal transplant recipients. Transplantation 2009; 87 (1): 112-7.

18. Israni AK, Snyder JJ, Skeans MA, Peng Y, Maclean JR, Weinhandl ED, et al. Predicting coronary heart disease after kidney transplantation: patient outcomes in renal transplantation (PORT) Study. Am J Transplant 2010; 10 (2): 338-53.

19. Thompson S, James M, Wiebe N, Hemmelgard B, Manns B, Klarenbach S, et al. Cause of death in patients with reduced kidney Function. J Am Soc Nephrol 2015; 26 (10): 2504-11. 
20. Meier-Kriesche H-U, Baliga R, Kaplan B. Decreased renal function is a strong risk factor for cardiovascular death after renal transplantation. Transplantation 2003; 75 (8): 1291-5.

21. Sarnak MJ, Levey AS. Cardiovascular disease and chronic renal disease: a new paradigm. Am J Kidney Dis 2000; 35 (4 Suppl 1): S117-31.

22. Liefeldt L, Budde K. Risk factors for cardiovascular disease in renal transplant recipients and strategies to minimize risk. Transpl Int 2010; 23 (12): 1191-204.

23. Prakash J, Ghosh B, Singh S, Soni A, Rathore SS. Causes of death in renal transplant recipients with functioning allograft. Indian J Nephrol 2012; 22 (4): 264-8.

24. Mourad G, Dussol B, Daugas E, Joly D, Juillard L, Henri $\mathrm{P}$, et al. Is renal allograft dysfunction a risk factor for severe infection in kidney transplant recipients? Transplant Proc 2012; 44 (9): 2821-3.
25. Webster AC, Craig JC, Simpson JM, Jones MP, Chapman JR. Identifying High Risk Groups and Quantifying Absolute Risk of Cancer After Kidney Transplantation: A Cohort Study of 15183 Recipients. Am J Transplant 2007; 7 (9): 2140-51.

26. Mazuecos A, Muñoz Terol JM, García Alvárez T, Sola E, Rodrígez Benot A, Dsuna A, et al. Increase in malignancies as cause of death in renal transplant patients. Transplant Proc 2009; 41 (6): 2159-62.

27. Lorenz EC, El-Zoghby ZM, Amer H, Dean PG, Hathcock MA, Kremers WK, et al. Kidney allograft function and histology in recipients dying with a functioning graft. Am J Transplant 2014; 14 (7): 1612-8.

28. Kasiske BL, Israni AK, Snyder JJ, Skeans MA, et al. The relationship between kidney function and long-term graft survival after kidney transplant. Am J Kidney Dis 2011; 57 (3): 466-75. 